\title{
OSCILLATION OF SUBLINEAR AND SUPERLINEAR SECOND ORDER NEUTRAL DIFFERENTIAL EQUATIONS
}

\author{
A.K. Tripathy ${ }^{1}$, A.K. Sethi ${ }^{2}$ \\ ${ }^{1,2}$ Department of Mathematics \\ Sambalpur University \\ Sambalpur, 768019, INDIA
}

\begin{abstract}
In this work, we establish the necessary and sufficient conditions for oscillation of a class of functional differential equations of the form:

$$
\left(r(t)(x(t)+p(t) x(\tau(t)))^{\prime}\right)^{\prime}+q(t) G(x(\sigma(t)))+v(t) H(x(\eta(t)))=0
$$
\end{abstract}

under the assumption that

$$
\int_{0}^{\infty} \frac{d t}{r(t)}=\infty
$$

for various ranges of $p(t)$.

AMS Subject Classification: $34 \mathrm{~K}, 34 \mathrm{C} 10$

Key Words: oscillation, nonoscillation, neutral, delay, nonlinear

\section{Introduction}

Consider the nonlinear neutral delay differential equations of the form:

$$
\left(r(t)(x(t)+p(t) x(\tau(t)))^{\prime}\right)^{\prime}+q(t) G(x(\sigma(t)))+v(t) H(x(\eta(t)))=0,
$$

where $r, q, v, \tau, \sigma, \eta \in C\left(\mathbb{R}_{+}, \mathbb{R}_{+}\right), p \in C\left(\mathbb{R}_{+}, \mathbb{R}\right)$ such that $\tau(t) \leq t, \sigma(t) \leq$ $t, \eta(t) \leq t$ with $\lim _{t \rightarrow \infty} \tau(t)=\infty=\lim _{t \rightarrow \infty} \sigma(t)=\infty=\lim _{t \rightarrow \infty} \eta(t)$ and $G, H \in$ $C(\mathbb{R}, \mathbb{R})$ satisfying the property $y G(y)>0, u H(u)>0$ for $y, u \neq 0$. The

Received: November 2, 2016

Revised: February 22, 2017

Published: February 28, 2017 (c) 2017 Academic Publications, Ltd. url: www.acadpubl.eu 
objective of this work is to establish the necessary and sufficient conditions for oscillation of solutions of (1) under the assumption

$$
\left(A_{0}\right) R(t)=\int_{0}^{t} \frac{d s}{r(s)} \rightarrow+\infty \text { as } t \rightarrow \infty,
$$

for various range of $p(t)$.

Baculikova et al. [4] have studied the linear counterpart of (1),

$$
\left(r(t)(x(t)+p(t) x(\tau(t)))^{\prime}\right)^{\prime}+q(t) x(\sigma(t))+v(t) x(\eta(t))=0
$$

when $0 \leq p(t) \leq p_{0}<\infty$. The authors have obtained sufficient conditions for oscillation of solutions of (2) through some comparison results, where the comparison results are unpredictable. In this work, an attempt is made to study the more general form of (2) without comparison results. It seems that our method is the next alternative to the work of [4], for any $|p(t)|<\infty$. Tripathy et al.[9] have studied (1) along with

$$
\left(r(t)(x(t)+p(t) x(\tau(t)))^{\prime}\right)^{\prime}+q(t) G(x(\sigma(t)))=0
$$

and obtained the sufficient conditions for oscillation, nonoscillation and asymptotic behavior of solutions of (1) and (3) provided $G, H$ could be linear or nonlinear. In this work, an attempt is to study (1) such that $G$ and $H$ could be strictly sublinear or superlinear. We note that not only the present work generalizes the work of [4], but also it generalizes the work of [2] and [3].

The neutral differential equations find numerous applications in natural sciences and technology. For instance, they are frequently used for the study of distributed networks containing lossless transmission lines (see for e.g [5]). In this paper, we restrict our attention to study (1) which includes a class of nonlinear functional differential equations of neutral type. In this direction we refer the reader to some of the works([1], [2], [3], [7], [8], [10], [11]) and the references cited therein.

Definition 1.1. By a solution of (1), we mean a continuously differentiable function $x(t)$ which is defined for $t \geq T^{*}=\min \left\{\tau\left(t_{0}\right), \sigma\left(t_{0}\right), \eta\left(t_{0}\right)\right\}$ such that $x(t)$ satisfies (1) for all $t \geq t_{0}$. In the sequel, it will always be assumed that the solutions of (1) exist on some half line $\left[t_{1}, \infty\right), t_{1} \geq t_{0}$. A solution of (1) is said to be oscillatory, if it has arbitrarily large zeros; otherwise, it is called non-oscillatory. Equation (1) is called oscillatory, if all its solutions are oscillatory. 


\section{Main Results}

This section deals with the necessary and sufficient conditions for oscillation of all solutions of (1). Throughout our discussion, we use the notation

$$
z(t)=x(t)+p(t) x(\tau(t))
$$

Lemma 2.1. Assume that $\left(A_{0}\right)$ holds. If $x(t)$ is a positive solution of (1) such that the corresponding function $z(t)>0$, then $z(t)$ satisfies $z(t)>$ $0, r(t) z^{\prime}(t)>0,\left(r(t) z^{\prime}(t)\right)^{\prime}<0$ eventually.

Proof. The proof of the lemma follows from [2] and hence the details are omitted.

Theorem 2.2. Let $-1<-a \leq p(t) \leq 0, a \geq 0, t \in \mathbb{R}_{+}$. Assume that $\left(A_{0}\right)$ holds. Furthermore, assume that

$\left(A_{1}\right) G(-u)=-G(u), H(-v)=-H(v), u, v \in \mathbb{R}$,

$\left(A_{2}\right) \quad \tau^{n}(t)=\tau^{n-1}(\tau(t))$ and $\lim _{n \rightarrow \infty} \tau^{n}(t)<\infty, t \in \mathbb{R}_{+}$

and

$\left(A_{3}\right) \quad G$ and $H$ are strictly sublinear satisfying the property

$$
\frac{G(u)}{u^{\beta}} \geq \frac{G(v)}{v^{\beta}}, \frac{H(u)}{u^{\beta}} \geq \frac{H(v)}{v^{\beta}}, 0<u \leq v, \beta<1
$$

hold. Then every unbounded solution of (1) oscillates if and only if $\left(A_{4}\right) \quad \int_{T}^{\infty}[q(s) G(C R(\sigma(s))+v(s) H(C R(\eta(s))] d s=\infty, T>0$ for every $C>0$.

Proof. On the contrary, let's assume that $x(t)$ be an unbounded nonoscillatory solution of (1) such that $x(t)>0$ for $t \geq t_{0}$. Hence, there exists $t_{1}>t_{0}$ such that

$$
x(t)>0, x(\tau(t))>0, x(\sigma(t))>0, x(\eta(t))>0 \text { for } t \geq t_{1} .
$$

Using (1), (1) becomes

$$
\begin{aligned}
\left(r(t) z^{\prime}(t)\right)^{\prime}= & -q(t) G(x(\sigma(t))-v(t) H(x(\eta(t)) \\
& \leq 0, \not \equiv 0 \text { for } t \geq t_{1} .
\end{aligned}
$$

Therefore, there exists $t_{2}>t_{1}$ such that $z(t), z^{\prime}(t)$ are of one sign on $\left[t_{2}, \infty\right)$. If there exists $t_{3}>t_{2}$ such that $z(t) \leq 0$ for $t \geq t_{3}$, then it follows that

$$
x(t) \leq x(\tau(t)) \leq x\left(\tau^{2}(t)\right) \leq x\left(\tau^{3}(t)\right) \leq \ldots \ldots . \leq x\left(t_{3}\right)
$$

implies that $x(t)$ is bounded, which is absurd. Hence, $z(t)>0$ and Lemma 2.1 holds for $t \geq t_{3}$. Using the fact that $\lim _{t \rightarrow \infty} r(t) z^{\prime}(t)$ exists and integrating $(2)$ 
from $t\left(>t_{3}\right)$ to $\infty$, we obtain

$$
\int_{t}^{\infty}[q(s) G(x(\sigma(s)))+v(s) H(x(\eta(s)))] d s \leq r(t) z^{\prime}(t)
$$

that is ,

$$
z^{\prime}(t) \geq \frac{1}{r(t)}\left[\int_{t}^{\infty} q(s) G(x(\sigma(s))) d s+\int_{t}^{\infty} v(s) H(x(\eta(s))) d s\right]
$$

for $t \geq t_{3}$. Let $t_{4}>t_{3}$ be such that

$$
R(t)-R\left(t_{4}\right) \geq \frac{1}{2} R(t), t \geq t_{4}
$$

Then integrating (3) from $t_{4}$ to $t\left(>t_{4}\right)$, we get

$$
\begin{aligned}
z(t)-z\left(t_{4}\right) & \geq \int_{t_{4}}^{t} \frac{1}{r(s)}\left[\int_{s}^{\infty} q(u) G(x(\sigma(u))) d u+\int_{s}^{\infty} v(u) H(x(\eta(u))) d u\right] d s \\
& \geq \int_{t_{4}}^{t} \frac{1}{r(s)}\left[\int_{t}^{\infty} q(u) G(x(\sigma(u))) d u+\int_{s}^{\infty} v(u) H(x(\eta(u))) d u\right] d s
\end{aligned}
$$

that is,

$$
\begin{gathered}
z(t) \geq\left(R(t)-R\left(t_{4}\right)\right) \int_{t}^{\infty}[q(u) G(x(\sigma(u)))+v(u) H(x(\eta(u)))] d u \\
\geq \frac{1}{2} R(t) \int_{t}^{\infty}[q(u) G(x(\sigma(u)))+v(u) H(x(\eta(u)))] d u, t \geq t_{4} .
\end{gathered}
$$

Using the fact that $r(t) z^{\prime}(t)$ is nonincreasing on $\left[t_{4}, \infty\right)$, we can find a constant $C>0$ and $t_{5}>t_{4}$ such that $r(t) z^{\prime}(t) \leq C$ for $t \geq t_{5}$ and hence $z(t) \leq$ $C R(t), t \geq t_{5} . G$ is strictly sublinear implies that

$$
\begin{aligned}
G(z(\sigma(t))) & =\frac{G(z(\sigma(t)))}{z^{\beta}(\sigma(t))} z^{\beta}(\sigma(t)) \\
& \geq \frac{G(C R(\sigma(t)))}{C^{\beta} R^{\beta}(\sigma(t))} z^{\beta}(\sigma(t)) .
\end{aligned}
$$

Consequently, (4) becomes

$$
z(t) \geq \frac{R(t)}{2} \int_{t}^{\infty}\left[\frac{q(u) G(C R(\sigma(u))) z^{\beta}(\sigma(u))}{C^{\beta} R^{\beta}(\sigma(u))}+\frac{v(u) H(C R(\eta(u))) z^{\beta}(\eta(u))}{C^{\beta} R^{\beta}(\eta(u))}\right] d u
$$


for $t \geq t_{5}$. If we define

$$
w(t)=\frac{1}{2} \int_{t}^{\infty}\left[\frac{q(u) G(C R(\sigma(u))) z^{\beta}(\sigma(u))}{C^{\beta} R^{\beta}(\sigma(u))}+\frac{v(u) H(C R(\eta(u))) z^{\beta}(\eta(u))}{C^{\beta} R^{\beta}(\eta(u))}\right] d u,
$$

then $z(t) \geq R(t) w(t)$ for $t \geq t_{5}$. Now,

$$
\begin{aligned}
w^{\prime}(t) & \leq-\frac{1}{2}\left[\frac{q(t) G(C R(\sigma(t))) z^{\beta}(\sigma(t))}{C^{\beta} R^{\beta}(\sigma(t))}+\frac{v(t) H(C R(\eta(t))) z^{\beta}(\eta(t))}{C^{\beta} R^{\beta}(\eta(t))}\right] \\
& \leq-\frac{1}{2}\left[\frac{q(t) G(C R(\sigma(t))}{C^{\beta}} w^{\beta}(\sigma(t))+\frac{v(t) H(C R(\eta(t)))}{C^{\beta}} w^{\beta}(\eta(t))\right] \\
& \leq 0, t \geq t_{5}
\end{aligned}
$$

implies that $w(t)$ is nonincreasing on $\left[t_{5}, \infty\right)$ and $\lim _{t \rightarrow \infty} w(t)$ exists. It is easy to verify that

$$
\begin{aligned}
{\left[w^{1-\beta}(t)\right]^{\prime} \leq } & -\frac{(1-\beta)}{2} w^{-\beta}(t) \\
& \times\left[\frac{q(t) G(C R(\sigma(t))}{C^{\beta}} w^{\beta}(\sigma(t))+\frac{v(t) H(C R(\eta(t)))}{C^{\beta}} w^{\beta}(\eta(t))\right] \\
\leq & -\frac{(1-\beta)}{2} w^{-\beta}(t)\left[\frac{q(t) G(C R(\sigma(t))}{C^{\beta}} w^{\beta}(t)+\frac{v(t) H(C R(\eta(t)))}{C^{\beta}} w^{\beta}(t)\right] \\
\leq & -\frac{(1-\beta)}{2 C^{\beta}}[q(t) G(C R(\sigma(t)))+v(t) H(C R(\eta(t)))]
\end{aligned}
$$

for $t \geq t_{5}$. Integrating (5) from $t_{5}$ to $t\left(>t_{5}\right)$, we obtain

$$
\begin{aligned}
\frac{(1-\beta)}{2 C^{\beta}} \int_{t^{5}}^{t}[q(s) G(C R(\sigma(s)))+v(s) H(C R(\eta(s)))] d s & \leq-\left[w^{1-\beta}(s)\right]_{t_{5}}^{t} \\
& <w^{1-\beta}\left(t_{5}\right)<\infty
\end{aligned}
$$

a contradiction to $\left(A_{4}\right)$.

Next, we suppose that $\left(A_{4}\right)$ doesn't hold. For $C>0$, let's assume that

$$
\int_{T}^{\infty}[q(t) G(C R(\sigma(t)))+v(t) H(C R(\eta(t)))] d t \leq \frac{C}{3} .
$$

Consider

$$
M=\left\{x: x \in C\left(\left[t_{0}, \infty\right), \mathbb{R}\right), x(t)=0 \text { for } t \in\left[t_{0}, T\right]\right.
$$

and

$$
\left.\frac{C}{3}[R(t)-R(T)] \leq x(t) \leq C[R(t)-R(T)]\right\}
$$


and define

$$
\Psi x(t)=\left\{\begin{array}{l}
0, \quad t \in\left[t_{0}, T\right] \\
-p(t) x(\tau(t))+\int_{T}^{t} \frac{1}{r(u)}\left[\frac{C}{3}+\int_{u}^{\infty} q(s) G(x(\sigma(s))) d s\right. \\
+\int_{u}^{\infty} v(s) H(x(\eta(s)) d s], t \geq T .
\end{array}\right.
$$

For every $x \in M$,

$$
\begin{aligned}
\Psi x(t) & \left.\geq \int_{T}^{t} \frac{1}{r(u)}\left[\frac{C}{3}+\int_{u}^{\infty}\{q(s) G x(\sigma(s)))+v(s) H(x(\eta(s)))\right\} d s\right] d u \\
& \geq \frac{C}{3} \int_{T}^{t} \frac{d u}{r(u)}=\frac{C}{3}[R(t)-R(T)]
\end{aligned}
$$

and $x(t) \leq C R(t)$ implies that

$$
\begin{aligned}
\Psi x(t) & \leq-p(t) x(\tau(t))+\frac{2 C}{3} \int_{T}^{t} \frac{d u}{r(u)} \\
& \leq a C[R(\tau(t))-R(T)]+\frac{2 C}{3}[R(t)-R(T)] \\
& \leq a C[R(t)-R(T)]+\frac{2 C}{3}[R(t)-R(T)] \\
& =\left(a+\frac{2}{3}\right) C[R(t)-R(T)] \\
& \leq C[R(t)-R(T)]
\end{aligned}
$$

implies that $\Psi x(t) \in M$. Define $u_{n}:\left[t_{0},+\infty\right) \rightarrow \mathbb{R}$ by the recursive formula

$$
u_{n}(t)=\left(\Psi u_{n-1}\right)(t), n \geq 1
$$

with the initial condition

$$
u_{0}(t)=\left\{\begin{array}{l}
0, \quad t \in\left[t_{0}, T\right] \\
\frac{C}{3}[R(t)-R(T)], t \geq T .
\end{array}\right.
$$

Inductively it is easy to verify that

$$
\frac{C}{3}[R(t)-R(T)] \leq u_{n-1}(t) \leq u_{n}(t) \leq C[R(t)-R(T)]
$$


for $t \geq T$. Therefore for $t \geq t_{0}, \lim _{n \rightarrow \infty} u_{n}(t)$ exists. By the Lebesgue's dominated convergence theorem, $u \in M$ and $(\Psi u)(t)=u(t)$, where $u(t)$ is a solution of (1) on $\left[t_{0}, \infty\right)$ such that $u(t)>0$. Hence, $\left(A_{6}\right)$ is necessary. This completes the proof of the theorem.

Theorem 2.3. Let $-1<-a \leq p(t) \leq 0, a>0$ for $t \in \mathbb{R}_{+}$. Assume that $\left(A_{0}\right),\left(A_{1}\right)$ and $\left(A_{2}\right)$ hold. Then every unbounded solution of $(1)$ oscillates if and only if $\left(A_{4}\right)$ holds for every $C>0$.

Proof. For the sufficient part, we proceed as in the proof of the Theorem 2.2 and obtain (2) for $t \geq t_{1}$. Since $z(t)$ is unbounded and monotonic increasing, then it follows that

$$
\lim _{t \rightarrow \infty} \frac{z(t)}{R(t)}=\lim _{t \rightarrow \infty} \frac{z^{\prime}(t)}{R^{\prime}(t)}=\lim _{t \rightarrow \infty} r(t) z^{\prime}(t)=\alpha<\infty .
$$

If $\alpha=0$, then $\lim _{t \rightarrow \infty} R(t)=+\infty$ implies that $\lim _{t \rightarrow \infty} z(t)<+\infty$, which is absurd (because $z(t)$ is unbounded). Hence $\alpha \neq 0$. Therefore, there exists a constant $C>0$ and a $t_{2}>t_{1}$ such that $z(t) \geq C R(t)$, for $t \geq t_{2}$. Using $z(t) \geq C R(t)$, for $t \geq t_{2}$ in (2) and then integrating from $t_{2}$ to $+\infty$, we obtain a contradiction to $\left(A_{4}\right)$ for every $C>0$. The necessary part is same as in Theorem 2.2. This completes the proof of the theorem.

Remark 2.4. In Theorem 2.3, $G$ and $H$ could be linear,sublinear or superlinear.

Theorem 2.5. Let $-1<-a \leq p(t) \leq 0, a>0, t \in \mathbb{R}_{+}$. Assume that $\left(A_{0}\right)-\left(A_{3}\right)$ hold. Then every solution of $(1)$ oscillates or converges to zero if and only if $\left(A_{4}\right)$ holds for every $C>0$.

Proof. Let $x(t)$ be a nonoscillatory solution of (1) such that $x(t)>0$ for $t \geq t_{0}$. Proceeding as in the proof of Theorem 2.2, we have (2) for $t \geq t_{1}$. Hence there exists $t_{2}>t_{1}$ such that $r(t) z^{\prime}(t)$ and $z(t)$ are of constant sign for $t \geq t_{2}$. The case $z(t)<0, r(t) z^{\prime}(t)<0$ follows from the proof of Theorem 2.2. It is easy to notice that the case $z(t)>0, r(t) z^{\prime}(t)<0$ is not possible (same as the former case, since $\left.\lim _{t \rightarrow \infty} z(t)<\infty\right)$. Assume that $z(t)<0, r(t) z^{\prime}(t)>0$ holds for $t \geq t_{2}$. Therefore,

$$
\begin{aligned}
0 \geq \lim _{t \rightarrow \infty} z(t) & =\limsup _{t \rightarrow \infty} z(t) \\
& \geq \limsup _{t \rightarrow \infty}(x(t)-a x(\tau(t))) \\
& \geq \limsup _{t \rightarrow \infty} x(t)+\liminf _{t \rightarrow \infty}(-a x(\tau(t)))
\end{aligned}
$$




$$
=(1-a) \limsup _{t \rightarrow \infty} x(t)
$$

implies that $\limsup _{t \rightarrow \infty} x(t)=0$ and hence $\lim _{t \rightarrow \infty} x(t)=0$.

The last case $z(t)>0, r(t) z^{\prime}(t)>0$ follows from Lemma 2.1. Hence, $\left(A_{4}\right)$ is a sufficient condition. The necessary case is same as in the Theorem 2.2. Thus, the proof of the theorem is complete.

Theorem 2.6. Let $-1<-a \leq p(t) \leq 0, a>0$, and $r(t) \geq r(\sigma(t))$ for $t \in \mathbb{R}_{+}$. Assume that $\left(A_{0}\right),\left(A_{1}\right)$ and $\left(A_{2}\right)$ hold. Furthermore, assume that $\eta(t) \geq \sigma(t)$,

$\left(A_{5}\right) \quad G$ is superlinear, that is, $\frac{G(u)}{u^{\beta}} \geq \frac{G(v)}{v^{\beta}}, u \geq v>0, \beta>1$

and

$\left(A_{6}\right) \quad H$ is superlinear, that $i s, \frac{H(u)}{u^{\beta}} \geq \frac{H(v)}{v^{\beta}}, u \geq v>0, \beta>1$

hold. Then every solution of (1) either oscillates or converges to zero if and only if

$\left(A_{7}\right) \quad \int_{0}^{\infty} \frac{1}{r(t)} \int_{t}^{\infty}[q(s)+L v(s)] d s d t=+\infty, L>0$, is a constant.

Proof. For the sufficient part, we use the same type of argument as in the proof of Theorem 2.5 for first three cases of the pair $z(t)$ and $r(t) z^{\prime}(t)$. Let's consider the cases $z(t)>0$ and $r(t) z^{\prime}(t)>0$ for $t \geq t_{1}$. So, there exists a constant $C>0$ and $t_{2}>t_{1}$ such that $z(\sigma(t)) \geq C, z(\eta(t)) \geq C$ for $t \geq t_{2}$. Consequently,

$$
\begin{aligned}
G(z(\sigma(t))) & =\frac{G(z(\sigma(t)))}{z^{\beta}(\sigma(t)} z^{\beta}(\sigma(t)) \\
& \geq \frac{G(C)}{C^{\beta}} z^{\beta}(\sigma(t))
\end{aligned}
$$

and $H(z(\eta(t))) \geq \frac{H(C)}{C^{\beta}} z^{\beta}(\eta(t))$, for $t \geq t_{2}$. Therefore, (3) becomes

$$
\begin{aligned}
r(t) z^{\prime}(t) & \geq \frac{G(C)}{C^{\beta}} \int_{t}^{\infty} q(s) z^{\beta}(\sigma(s)) d s+\frac{H(C)}{C^{\beta}} \int_{t}^{\infty} v(s) z^{\beta}(\eta(s)) d s, \\
& \geq\left[\frac{G(C)}{C^{\beta}} \int_{t}^{\infty} q(s) d s\right] z^{\beta}(\sigma(t))+\left[\frac{H(C)}{C^{\beta}} \int_{t}^{\infty} v(s) d s\right] z^{\beta}(\eta(t)),
\end{aligned}
$$

that is,

$$
r(\sigma(t)) z^{\prime}(\sigma(t)) \geq\left[\frac{G(C)}{C^{\beta}} \int_{t}^{\infty} q(s) d s+\frac{H(C)}{C^{\beta}} \int_{t}^{\infty} v(s) d s\right] z^{\beta}(\sigma(t))
$$


for $t \geq t_{2}$ implies that

$$
\begin{aligned}
z^{\prime}(\sigma(t)) & \geq\left[\frac{G(C)}{C^{\beta}} \int_{t}^{\infty} q(s) d s+\frac{H(C)}{C^{\beta}} \int_{t}^{\infty} v(s) d s\right] \frac{z^{\beta}(\sigma(t))}{r(\sigma(t))} \\
& \geq \frac{G(C)}{C^{\beta}} \frac{z^{\beta}(\sigma(t))}{r(t)} \int_{t}^{\infty}[q(s)+L v(s)] d s
\end{aligned}
$$

where $L=\frac{H(C)}{G(C)} \geq 0$. Integrating the above inequality from $t_{2}$ to $+\infty$, we get

$$
\frac{G(C)}{C^{\beta}} \int_{t_{2}}^{\infty} \frac{1}{r(t)} \int_{t}^{\infty}[q(s)+L v(s)] d s d t \leq \int_{t_{2}}^{\infty} \frac{z^{\prime}(\sigma(t))}{z^{\beta}(\sigma(t))} d t<\infty
$$

which is a contradiction to $\left(A_{7}\right)$.

Next, we show that $\left(A_{7}\right)$ is necessary. Assume that $\left(A_{7}\right)$ fails to hold and let

$$
G(C) \int_{T}^{t} \frac{1}{r(s)} \int_{s}^{\infty}[q(\theta)+L v(\theta)] d \theta d s \leq \frac{C}{3}, T \geq T^{*},
$$

where $C>0$ is a constant. Consider

$$
M=\left\{x \in C\left(\left[t_{0}, \infty\right), \mathbb{R}\right): x(t)=\frac{C}{3}, t \in\left[t_{0}, T\right] ; \frac{C}{3} \leq x(t) \leq C, \text { for } t \geq T\right\},
$$

and define

$$
\Psi x(t)=\left\{\begin{array}{l}
\frac{C}{3}, \quad t \in\left[t_{0}, T\right] \\
-p(t) x(\tau(t))+\frac{C}{3} \\
+\int_{T}^{t} \frac{1}{r(s)} \int_{s}^{\infty}[q(\theta) G(x(\sigma(\theta)))+v(\theta) H(x(\eta(\theta)))] d \theta d s, t \geq T
\end{array}\right.
$$

for every $x \in M, \Psi x(t) \geq \frac{C}{3}$ and

$$
\begin{aligned}
\psi x(t) & \leq a C+\frac{C}{3}+\int_{T}^{t} \frac{1}{r(s)} \int_{s}^{\infty}[q(\theta) G(C)+v(\theta) H(C)] d \theta d s \\
& =a C+\frac{C}{3}+G(C) \int_{T}^{t} \frac{1}{r(s)} \int_{s}^{\infty}[q(\theta)+L v(\theta)] d \theta d s \\
& \leq a C+\frac{C}{3}+\frac{C}{3}=\left(a+\frac{2}{3}\right) C \\
& \leq C
\end{aligned}
$$

implies that $\Psi x \in M$. The rest of the proof follows from Theorem 2.2 and Theorem 2.5. This completes the proof of the theorem. 
Theorem 2.7. Let $0 \leq p(t) \leq a<1$ and $r(t) \geq r(\sigma(t)), \eta(t) \geq \sigma(t)$ for $t \in \mathbb{R}_{+}$. Assume that $\left(A_{0}\right),\left(A_{1}\right),\left(A_{5}\right)$ and $\left(A_{6}\right)$ hold. Furthermore, assume that $G$ and $H$ are Lipschitzian on the the interval of the form $[c, d], 0<c<$ $d<\infty$. Then every solution of (1) oscillates if and only if $\left(A_{7}\right)$ holds.

Proof. Let $x(t)$ be a nonoscillatory solution of (1). Then proceeding as in Theorem 2.2 and due to Lemma 2.1, we obtain that $r(t) z^{\prime}(t)>0$, for $t \geq t_{1}$. Therefore, there exists $t_{2}>t_{1}$ such that $z(t)$ is nondecreasing for $t \geq t_{2}$. So, we can find $t_{3}>t_{2}$ and $C>0$ such that $z(t) \geq C$ for $t \geq t_{3}$. Since

$$
\begin{aligned}
z(t)-p(t) z(\tau(t)) & =x(t)+p(t) x(\tau(t))-p(t) x(\tau(t)) \\
& -p(t) p(\tau(t)) p(\tau(\tau(t))) \\
& =x(t)-p(t) p(\tau(t)) p(\tau(\tau(t))) \\
& \leq x(t)
\end{aligned}
$$

then $x(t) \geq(1-a) z(t)$ and hence (1) becomes

$$
\left(r(t) z^{\prime}(t)\right)^{\prime}+q(t) G(z(\sigma(t)))+v(t) H(z(\eta(t))) \leq 0,
$$

for $t \geq t_{3}$. Using $\left(A_{5}\right)$ and $\left(A_{6}\right)$ it follows that

$$
\begin{aligned}
G((1-a) z(\sigma(t))) & =\frac{G((1-a) z(\sigma(t)))}{(1-a)^{\beta} z^{\beta}(\sigma(t))}(1-a)^{\beta} z^{\beta}(\sigma(t)) \\
& \geq \frac{G(C(1-a))}{C^{\beta}(1-a)^{\beta}}(1-a)^{\beta} z^{\beta}(\sigma(t))
\end{aligned}
$$

and

$$
H((1-a) z(\eta(t))) \geq \frac{H(C(1-a))}{C^{\beta}(1-a)^{\beta}}(1-a)^{\beta} z^{\beta}(\eta(t)) .
$$

The remaining portion of the sufficient part follows from Theorem2.6.

Conversely, suppose that $\left(A_{7}\right)$ fails to hold. Then there exists $T \geq T^{*}$ such that

$$
\int_{T}^{\infty} \frac{1}{r(s)} \int_{s}^{\infty}[q(\theta)+L v(\theta)] d \theta d s<\frac{1-a}{5 K},
$$

where $K=\max \left\{K_{1}, \frac{K_{2}}{L}, G(1)\right\}, K_{1}$ is the Lipschitz constant of $G$ and $K_{2}$ is the Lipschitz constant of $H$ on $\left[\frac{7(1-a)}{10}, 1\right]$ for $t \geq t_{0}$. Let $X=B C\left(\left[t_{0}, \infty\right), \mathbb{R}\right)$ be the space of real valued continuous functions on $\left[t_{0}, \infty\right)$. Indeed, $X$ is a Banach space with respect to sup norm defined by

$$
\|x\|=\sup \left\{|x(t)|: t \geq t_{0}\right\}
$$


Define

$$
S=\left\{u \in X: \frac{7(1-a)}{10} \leq u(t) \leq 1, t \geq t_{0}\right\}
$$

We notice that $S$ is a closed convex subspace of $X$. Let $\Psi: S \rightarrow S$ be such that

$$
\Psi x(t)=\left\{\begin{array}{l}
\Psi x(T), \quad t \in\left[t_{0}, T\right] \\
-p(t) x(\tau(t))+\frac{9+a}{10} \\
-\int_{t}^{\infty} \frac{1}{r(s)} \int_{s}^{\infty}[q(\theta) G(x(\sigma(\theta)))+v(\theta) H(x(\eta(\theta))] d \theta d s, t \geq T .
\end{array}\right.
$$

For every $x \in X, \Psi x(t) \leq \frac{9+a}{10} \leq 1$ and

$$
\Psi x(t) \geq-a+\frac{9+a}{10}-\frac{1-a}{5}=\frac{7}{10}(1-a)
$$

implies that $\Psi x \in S$. Now for $x_{1}, x_{2} \in S$, we have

$$
\begin{aligned}
\left|\Psi x_{1}(t)-\Psi x_{2}(t)\right| & \leq a\left|x_{1}(\tau(t))-x_{2}(\tau(t))\right| \\
& +\int_{t}^{\infty} \frac{1}{r(s)} \int_{s}^{\infty} q(\theta)\left|G\left(x_{1}(\sigma(\theta))\right)-G\left(x_{2}(\sigma(\theta))\right)\right| d \theta d s \\
& +\int_{t}^{\infty} \frac{1}{r(s)} \int_{s}^{\infty} v(\theta)\left|H\left(x_{1}(\eta(\theta))\right)-H\left(x_{2}(\eta(\theta))\right)\right| d \theta d s
\end{aligned}
$$

that is,

$$
\begin{aligned}
\left|\Psi x_{1}(t)-\Psi x_{2}(t)\right| & \leq a\left\|x_{1}-x_{2}\right\|+\left\|x_{1}-x_{2}\right\| K_{1} \int_{t}^{\infty} \frac{1}{r(s)} \int_{s}^{\infty} q(\theta) d \theta d s \\
& +\left\|x_{1}-x_{2}\right\|+K_{2} \int_{t}^{\infty} \frac{1}{r(s)} \int_{s}^{\infty} v(\theta) d \theta d s \\
& \leq\left(a+\frac{1-a}{5}\right)\left\|x_{1}-x_{2}\right\| \\
& =\frac{1+4 a}{5}\left\|x_{1}-x_{2}\right\|
\end{aligned}
$$

Therefore, $\left\|\Psi x_{1}-\Psi x_{2}\right\| \leq \frac{1+4 a}{5}\left\|x_{1}-x_{2}\right\|$ implies that $\Psi$ is a contraction. By using Banach's fixed point theorem, it follows that $\Psi$ has a unique fixed point $x(t)$ in $\left[\frac{7(1-a)}{10}, 1\right]$. Hence, $\left(A_{7}\right)$ is the necessary condition for oscillation of $(1)$. This completes the proof of the theorem.

Theorem 2.8. Let $0 \leq p(t) \leq a<1$, for $t \in \mathbb{R}_{+}$. Assume that $\left(A_{0}\right)$ and $\left(A_{1}\right)$ hold. Furthermore, assume that $G$ and $H$ are Lipschitzian on the intervals of the form $[c, d], 0<c<d<\infty$. Then every bounded solutions of (1) oscillates if and only if $\left(A_{7}\right)$ holds. 
Proof. Sufficient part is an immediate integration following Theorem 2.7 and the necessary part is same as in the proof of Theorem 2.7. Hence the details are omitted. Thus the proof of theorem is complete.

Theorem 2.9. Let $1 \leq p(t) \leq a<\infty, G(a) \geq H(a)$ and $r(t) \geq r(\sigma(t))$ for $t \in \mathbb{R}_{+}$. Assume that $\sigma(t) \leq \tau(t)$ for $t \in \mathbb{R}_{+}$and $\left(A_{0}\right),\left(A_{1}\right),\left(A_{3}\right)$ hold. Furthermore, assume that $\left(A_{8}\right)$ there exist $\lambda, \mu>0$ such that $G(u)+G(s) \geq \lambda G(u+s), H(u)+H(s) \geq \lambda H(u+s)$ for $u, s \in \mathbb{R}_{+}$, (see for e.g $[6]$ ), $\left(A_{9}\right) G(u s) \leq G(u) G(s), H(u s) \leq H(u) H(s), u, s \in \mathbb{R}_{+}$, $\left(A_{10}\right) \quad \tau o \sigma=\sigma o \tau, \tau o \eta=\eta o \tau$ for all $t \in \mathbb{R}_{+}$ and $\left(A_{11}\right) \quad \int_{T}^{\infty}[Q(s) G(C R(\sigma(s)))+V(s) H(C R(\eta(s)))] d s=\infty, T>0$ hold for every $C>0$, where $Q(t)=\min \{q(t), q(\tau(t))\}, V(t)=\min \{v(t), v(\tau(t))\}$, then every solution of (1) oscillates.

Proof. Proceeding as in the proof of Theorem 2.7, we conclude that $r(t) z^{\prime}(t)>$ 0 for $t \geq t_{1}$ and $\lim _{t \rightarrow \infty} r(t) z^{\prime}(t)$ exists. From (1) it is easy to see that

$$
\begin{aligned}
0 & =\left(r(t) z^{\prime}(t)\right)^{\prime}+q(t) G(x(\sigma(t)))+v(t) H(x(\eta(t))) \\
& \left.+G(a)\left[r(\tau(t)) z^{\prime}(\tau(t))\right)^{\prime}+q(\tau(t))\right) G(x(\sigma(\tau(t)))+v(\tau(t))) H(x(\eta(\tau(t)))] .
\end{aligned}
$$

Using $\left(A_{8}\right),\left(A_{9}\right)$ and $\left(A_{10}\right)$ in the above relation, it follows that

$$
\begin{aligned}
0 & \geq\left(r(t) z^{\prime}(t)\right)^{\prime}+G(a)\left(r(\tau(t)) z^{\prime}(\tau(t))\right)^{\prime}+\lambda Q(t) G(z(\sigma(t)))+v(t) H(x(\eta(t))) \\
& +G(a) v(\tau(t)) H(x(\eta(\tau(t))) \\
& \geq\left(r(t) z^{\prime}(t)\right)^{\prime}+G(a)\left(r(\tau(t)) z^{\prime}(\tau(t))\right)^{\prime}+\lambda Q(t) G(z(\sigma(t))) \\
& +v(t) H(x(\eta(t)))+H(a) v(\tau(t)) H(x(\eta(\tau(t))) \\
& \geq\left(r(t) z^{\prime}(t)\right)^{\prime}+G(a)\left(r(\tau(t)) z^{\prime}(\tau(t))\right)^{\prime}+\lambda Q(t) G(z(\sigma(t)))+\mu V(t) H(z(\eta(t))) .
\end{aligned}
$$

Therefore, integrating the last inequality from $t\left(>t_{1}\right)$ to $+\infty$, we obtain

$$
\begin{aligned}
\lambda \int_{t}^{\infty} Q(s) G(z(\sigma(s))) d s & +\mu \int_{t}^{\infty} V(s) H(z(\eta(s))) d s \\
\leq & r(t) z^{\prime}(t)+G(a)\left(r(\tau(t)) z^{\prime}(\tau(t))\right. \\
& \leq r(t) z^{\prime}(t)+G(a) r(\sigma(t)) z^{\prime}(\sigma(t)) \\
& \leq(1+G(a)) r(\sigma(t)) z^{\prime}(\sigma(t)) .
\end{aligned}
$$

Consequently, for $t \geq t_{2}>t_{1}$

$$
z^{\prime}(\sigma(t)) \geq \frac{1}{(1+G(a)) r(\sigma(t))}
$$




$$
\begin{array}{r}
{\left[\lambda \int _ { t } ^ { \infty } Q ( s ) G \left(z(\sigma(s)) d s+\mu \int_{t}^{\infty} V(s) H(z(\eta(s)) d s]\right.\right.} \\
\geq \frac{1}{(1+G(a)) r(t)}\left[\lambda \int _ { t } ^ { \infty } Q ( s ) G \left(z(\sigma(s)) d s+\mu \int_{t}^{\infty} V(s) H(z(\eta(s)) d s] .\right.\right.
\end{array}
$$

Proceeding as in the proof of Theorem 2.2, we find (4) as follows:

$$
\begin{aligned}
\frac{z(\sigma(t))}{R(t)} \geq & \frac{C^{-\beta}}{2(1+G(a))}\left[\lambda \int_{t}^{\infty} Q(s) G(C R(\sigma(s)))\left\{\frac{z(\sigma(s))}{R(\sigma(s))}\right\}^{\beta} d s\right. \\
& \left.+\mu \int_{t}^{\infty} V(s) H(C R(\sigma(s)))\left\{\frac{z(\eta(s))}{R(\eta(s))}\right\}^{\beta} d s\right]
\end{aligned}
$$

that is,

$$
\begin{aligned}
\frac{z(\sigma(t))}{R(\sigma(t))} \geq & \frac{C^{-\beta}}{2(1+G(a))}\left[\lambda \int_{t}^{\infty} Q(s) G(C R(\sigma(s)))\left\{\frac{z(\sigma(s))}{R(\sigma(s))}\right\}^{\beta} d s\right. \\
& \left.+\mu \int_{t}^{\infty} V(s) H(C R(\sigma(s)))\left\{\frac{z(\eta(s))}{R(\eta(s))}\right\}^{\beta} d s\right]
\end{aligned}
$$

for $t \geq t_{2}$. The rest part of the proof follows from the proof of Theorem 2.2. This completes the proof of the theorem.

Theorem 2.10. Let $1 \leq p(t) \leq a<\infty, G(a) \geq H(a)$ and $r(t) \geq r(\sigma(t)$ for $t \in \mathbb{R}_{+}$. Assume that $\sigma(t) \leq \tau(t)$ for $t \in \mathbb{R}_{+}$, and $\left(A_{0}\right),\left(A_{1}\right),\left(A_{5}\right),\left(A_{6}\right)$, $\left(A_{8}\right),\left(A_{9}\right)$ hold. If $\left(A_{12}\right) \tau(t)$ is bijective

and

$\left(A_{13}\right) \quad \int_{0}^{\infty} \frac{1}{r(t)}\left[\int_{t}^{\infty} Q(s) d s+L \int_{t}^{\infty} V(s) d s\right] d t=+\infty$,

where $Q(t)$ and $V(t)$ are defined in Theorem 2.9, then every solution of (1) oscillates.

Proof. The proof of the theorem follows from (7) and the Theorem 2.6. Hence, the details are omitted.

Theorem 2.11. Let $1<a_{1} \leq p(t) \leq a_{2}<\infty, a_{1}^{2} \geq a_{2}$ for $t \in \mathbb{R}_{+}$. Let $G$ and $H$ be Lipschitzian on the intervals of the form $[c, d], 0<c<d<\infty$. If

$$
\int_{0}^{\infty} \frac{1}{r(t)}\left[\int_{t}^{\infty} q(s) d s+L \int_{t}^{\infty} v(s) d s\right] d t<\infty, L>0
$$

then (1) admits a positive bounded solution. 
Proof. If possible, let there exist $T \geq T^{*}$ such that

$$
\int_{T}^{\infty} \frac{1}{r(t)}\left[\int_{t}^{\infty} q(s) d s+L \int_{t}^{\infty} v(s) d s\right] d t<\frac{a_{1}-1}{3 K}
$$

where $K=\max \left\{K_{1}, \frac{K_{2}}{L}, G(d)\right\}, K_{1}$ is the Lipschitz constant of $G$ and $K_{2}$ is the Lipschitz constant of $H$ on $[c, d]$ with

$$
\begin{gathered}
c=\frac{3 \mu\left(a_{1}^{2}-a_{2}\right)-a_{2}\left(a_{1}-1\right)}{3 a_{1}^{2} a_{2}} \\
d=\frac{a_{1}-1+3 \mu}{3 a_{1}}, \mu>\frac{a_{2}\left(a_{1}-1\right)}{3\left(a_{1}^{2}-a_{2}\right)}>0 .
\end{gathered}
$$

Let $X=B C\left(\left[t_{0}, \infty\right), \mathbb{R}\right)$ be the space of real valued continuous functions on $\left[t_{0}, \infty\right)$. Indeed, $X$ is a Banach space with respect to the sup norm defined by

$$
\|x\|=\operatorname{Sup}\left\{|x(t)|: t \geq t_{0}\right\}
$$

Define

$$
S=\left\{u \in X: c \leq u(t) \leq d, t \geq t_{0}\right\}
$$

We may note that $S$ is a closed convex subspace of $X$. Let $\Psi: S \rightarrow S$ be such that

$$
\Psi x(t)=\left\{\begin{array}{l}
\Psi x(T), \quad t \in\left[T_{0}, T\right] \\
-\frac{x\left(\tau^{-1}(t)\right)}{p\left(\tau^{-1}(t)\right)}+\frac{\mu}{p\left(\tau^{-1}(t)\right)} \\
+\frac{1}{p\left(\tau^{-1}(t)\right)} \int_{T}^{\left.\tau^{-1}(t)\right)} \frac{1}{r(s)}\left[\int_{s}^{\infty} q(\theta) G(x(\sigma(\theta))) d \theta\right. \\
\left.+\int_{s}^{\infty} v(\theta) H(x(\eta(\theta))) d \theta\right] d s, t \geq T .
\end{array}\right.
$$

For every $x \in S$,

$$
\begin{aligned}
\Psi x(t) & \leq \frac{\mu}{p\left(\tau^{-1}(t)\right)}+\frac{1}{p\left(\tau^{-1}(t)\right)} \int_{T}^{\left.\tau^{-1}(t)\right)} \frac{G(d)}{r(s)}\left[\int_{s}^{\infty} q(\theta) d \theta+\frac{H(d)}{G(d)} \int_{s}^{\infty} v(\theta) d \theta\right] d s \\
& \leq \frac{\mu}{p\left(\tau^{-1}(t)\right)}+\frac{1}{a_{1}} \int_{T}^{\infty} \frac{G(d)}{r(s)}\left[\int_{s}^{\infty} q(\theta) d \theta+L \int_{s}^{\infty} v(\theta) d \theta\right] d s \\
& \leq \frac{\mu}{a_{1}}+\frac{G(d)}{a_{1}} \int_{T}^{\infty} \frac{1}{r(s)}\left[\int_{s}^{\infty} q(\theta) d \theta+L \int_{s}^{\infty} v(\theta) d \theta\right] d s \\
& \leq \frac{1}{a_{1}}\left[\frac{a_{1}-1}{3}+\mu\right]=b
\end{aligned}
$$


and

$$
\begin{aligned}
\Psi x(t) & \geq-\frac{x\left(\tau^{-1}(t)\right)}{p\left(\tau^{-1}(t)\right)}+\frac{\mu}{p\left(\tau^{-1}(t)\right)} \\
& >-\frac{d}{a_{1}}+\frac{\mu}{a_{2}}=c
\end{aligned}
$$

implies that $\Psi x \in S$. Again for $x_{1}, x_{2} \in S$

$$
\begin{aligned}
\mid \Psi x_{1}(t) & -\Psi x_{2}(t)\left|\leq \frac{1}{\left|p\left(\tau^{-1}(t)\right)\right|}\right| x_{1}\left(\tau^{-1}(t)\right)-x_{2}\left(\tau^{-1}(t)\right) \mid \\
& +\frac{K_{1}}{\left|p\left(\tau^{-1}(t)\right)\right|} \int_{T}^{\left.\tau^{-1}(t)\right)} \frac{1}{r(s)} \int_{s}^{\infty} q(\theta)\left|x_{1}(\sigma(\theta))-x_{2}(\sigma(\theta))\right| d \theta d s \\
& +\frac{K_{2}}{\left|p\left(\tau^{-1}(t)\right)\right|} \int_{T}^{\left.\tau^{-1}(t)\right)} \frac{1}{r(s)} \int_{s}^{\infty} v(\theta)\left|x_{1}(\eta(\theta))-x_{2}(\eta(\theta))\right| d \theta d s \\
\leq & \frac{1}{a_{1}}\left\|x_{1}-x_{2}\right\|+\frac{K_{1}}{a_{1}}\left\|x_{1}-x_{2}\right\| \int_{T}^{\infty} \frac{1}{r(s)} \int_{s}^{\infty} q(\theta) d \theta d s \\
& +\frac{K_{2}}{a_{1}}\left\|x_{1}-x_{2}\right\| \int_{T}^{\infty} \frac{1}{r(s)} \int_{s}^{\infty} v(\theta) d \theta d s \\
\leq & \frac{1}{a_{1}}\left(1+\frac{a_{1}-1}{3}\right)\left\|x_{1}-x_{2}\right\|
\end{aligned}
$$

implies that

$$
\left\|\Psi x_{1}-\Psi x_{2}\right\| \leq\left(\frac{1}{a_{1}}+\frac{a_{1}-1}{3 a_{1}}\right)\left\|x_{1}-x_{2}\right\| .
$$

Since $\left(\frac{1}{a_{1}}+\frac{a_{1}-1}{3 a_{1}}\right)<1$, then $\Psi: S \rightarrow S$ is a contraction. By the Banach's fixed point theorem, $\Psi$ has a unique fixed point on $[c, d]$. It is easy to verify that

$$
x(t)=\left\{\begin{array}{l}
\Psi x(T), \quad t \in\left[t_{0}, T\right] \\
-\frac{x\left(\tau^{-1}(t)\right)}{p\left(\tau^{-1}(t)\right)}+\frac{\mu}{p\left(\tau^{-1}(t)\right)} \\
+\frac{1}{p\left(\tau^{-1}(t)\right)} \int_{T}^{\left.\tau^{-1}(t)\right)} \frac{1}{r(s)}\left[\int_{s}^{\infty} q(\theta) G(x(\sigma(\theta))) d \theta\right. \\
\left.+\int_{s}^{\infty} v(\theta) H(x(\sigma(\theta))) d \theta\right] d s, t \geq T
\end{array}\right.
$$

is a positive bounded solution of $(1)$ on $[c, d]$. Hence, the proof of the theorem is complete.

Theorem 2.12. Let $-\infty<-a_{1} \leq p(t) \leq-a_{2}<-1$ for $t \in \mathbb{R}_{+}$, where $a_{1}, a_{2}>0$ such that $3 a_{2}>a_{1}$. Assume that $\left(A_{0}\right),\left(A_{1}\right)$ and $\left(A_{12}\right)$ hold. 
Furthermore, assume that $G$ and $H$ are Lipschitzian on the intervals of the form $[c, d], 0<c<d<\infty$. Then every bounded solution of (1) oscillates or tends to zero if and only if $\left(A_{7}\right)$ holds.

Proof. Let $x(t)$ be a nonoscillatory solution of (1) which is bounded. Then proceeding as in Theorem 2.2, it follows that $z(t), r(t) z^{\prime}(t)$ are monotonic functions on $\left[t_{2}, \infty\right)$. Since $x(t)$ is bounded, then by $(1), z(t)$ is bounded and hence $\lim _{t \rightarrow \infty} z(t)$ exists. It is easy to see that the case $z(t)<0, r(t) z^{\prime}(t)<0$ is not possible. Using the proof of Lemma 2.1, we conclude that the case $z(t)>0, r(t) z^{\prime}(t)<0$ doesn't arise. Therefore, we consider the cases $z(t)>$ $0, r(t) z^{\prime}(t)>0$ and $z(t)<0, r(t) z^{\prime}(t)>0$ on $\left[t_{3}, \infty\right), t_{3}>t_{2}$. With the former case, we can find a constant $C>0$ and $t_{4}>t_{3}$ such that $z(\sigma(t) \geq C, z(\eta(t) \geq C$ for $t \geq t_{4}$, that is, $y(\sigma(t)) \geq z(\sigma(t)) \geq C$ and $y(\eta(t)) \geq z(\eta(t)) \geq C$ for $t \geq t_{4}$. Hence, (1) becomes

$$
\left(r(t) z^{\prime}(t)\right)^{\prime}+G(C) q(t)+H(C) v(t) \leq 0, t \geq t_{4}
$$

Integrating the above inequality from $t\left(>t_{4}\right)$ to $+\infty$, we obtain

$$
G(C) \int_{t}^{\infty} q(s) d s+H(C) d s \leq r(t) z^{\prime}(t)
$$

that is,

$$
\int_{t_{4}}^{\infty} \frac{G(C)}{r(t)} \int_{t}^{\infty}[q(s)+L v(s)] d s d t<\infty, L=\frac{H(C)}{G(C)},
$$

a contradiction to $\left(A_{7}\right)$. For the latter case, we claim that $\lim _{t \rightarrow \infty} z(t)=0$. If not, there exist $\alpha<0$ and $t_{4}>t_{3}$ such that $z\left(\tau^{-1}(\sigma(t))\right)<\alpha, z\left(\tau^{-1}(\eta(t))\right)<\alpha$ for $t \geq t_{4}$. Hence, $z(t) \geq-a_{1} x(\tau(t))$ implies that $x(t) \geq-a_{1}^{-1} z\left(\tau^{-1}(t)\right)$, that is, $x(\sigma(t)) \geq-a_{1}^{-1} z\left(\tau^{-1}(\sigma(t))\right) \geq-a_{1}^{-1} \alpha$ and $z(\eta(t)) \geq-a_{1}^{-1} z\left(\tau^{-1}(\eta(t))\right) \geq$ $-a_{1}^{-1} \alpha$ for $t \geq t_{4}$. Consequently, (1) reduces to

$$
\left(r(t) z^{\prime}(t)\right)^{\prime}+G\left(-a_{1}^{-1} \alpha\right) q(t)+H\left(-a_{1}^{-1} \alpha\right) v(t) \leq 0,
$$

for $t \geq t_{4}$. Using the same type of argument as in the former case, we get a contradiction to $\left(A_{7}\right)$. Thus, our claim holds and hence

$$
\begin{aligned}
0=\lim _{t \rightarrow \infty} z(t) & =\liminf _{t \rightarrow \infty}(x(t)+p(t) x(\tau(t))) \\
& \leq \liminf _{t \rightarrow \infty}\left(x(t)-a_{2} x(\tau(t))\right) \\
& \leq \limsup _{t \rightarrow \infty} x(t)+\liminf _{t \rightarrow \infty}\left(-a_{2} x(\tau(t))\right)
\end{aligned}
$$




$$
=\left(1-a_{2}\right) \limsup _{t \rightarrow \infty} x(t)
$$

implies that $\limsup _{t \rightarrow \infty} x(t)=0$ because $\left.1-a_{2}<0\right]$. Therefore, $\lim _{t \rightarrow \infty} x(t)=0$.

For the necessary part, it is possible to find $T \geq T^{*}$ such that

$$
\int_{T}^{\infty} \frac{1}{r(s)} \int_{s}^{\infty}[q(t)+L v(t)] d t d s<\frac{a_{2}-1}{3 K}
$$

where $K=\max \left\{K_{1}, \frac{K_{2}}{L}, G(1)\right\}, \quad K_{1}$ and $K_{2}$ are Lipschitz constants of $G$ and $H$ on $[a, 1]$ respectively, where $a=\frac{\left(a_{2}-1\right)\left(3 a_{2}-a_{1}\right)}{3 a_{1} a_{2}}$. Let $X=B C\left(\left[t_{0}, \infty\right), \mathbb{R}\right)$ be the space of real valued continuous functions defined on $\left[t_{0}, \infty\right)$. Indeed, $X$ is a Banach space with the sup norm defined by

$$
\|x\|=\sup \left\{|x(t)|: t \geq t_{0}\right\}
$$

Define

$$
S=\left\{u \in X: a \leq u(t) \leq 1, t \geq t_{0}\right\}
$$

and we note that $S$ is a closed convex subspace of $X$. Let $\Psi: S \rightarrow S$ be such that

$$
\Psi x(t)=\left\{\begin{array}{l}
\psi x(T), \quad t \in\left[t_{0}, T\right] \\
-\frac{x\left(\tau^{-1}(t)\right)}{p\left(\tau^{-1}(t)\right)}-\frac{a_{2}-1}{p\left(\tau^{-1}(t)\right)}+\frac{1}{p\left(\tau^{-1}(t)\right)} \int_{T}^{\tau^{-1}(t)} \frac{1}{r(s)}\left[\int_{s}^{\infty} q(\theta) G(x(\sigma(\theta))) d \theta\right. \\
\left.+\int_{s}^{\infty} v(\theta) H(x(\eta(\theta))) d \theta\right] d s, t \geq T .
\end{array}\right.
$$

For every $x \in S$,

$$
\Psi x(t) \leq-\frac{x\left(\tau^{-1}(t)\right)}{p\left(\tau^{-1}(t)\right)}-\frac{a_{2}-1}{p\left(\tau^{-1}(t)\right)} \leq \frac{1}{a_{2}}+\frac{a_{2}-1}{a_{2}}=1
$$

and

$$
\begin{aligned}
\Psi x(t) & \geq-\frac{a_{2}-1}{p\left(\tau^{-1}(t)\right)}+\frac{1}{p\left(\tau^{-1}(t)\right)} \int_{T}^{\tau^{-1}(t)} \frac{1}{r(s)}\left[\int_{s}^{\infty} q(\theta) G(x(\sigma(\theta))) d \theta\right. \\
& \left.+\int_{s}^{\infty} v(\theta) H(x(\eta(\theta))) d \theta\right] d s \\
& \geq-\frac{a_{2}-1}{a_{1}}+\frac{G(1)}{p\left(\tau^{-1}(t)\right)} \int_{T}^{\tau^{-1}(t)} \frac{1}{r(s)}\left[\int_{s}^{\infty} q(\theta) d \theta+\frac{H(1)}{G(1)} \int_{s}^{\infty} v(\theta) d \theta\right] d s \\
& \geq-\frac{a_{2}-1}{a_{1}}-\frac{G(1)}{a_{2}} \int_{T}^{\infty} \frac{1}{r(s)}\left[\int_{s}^{\infty} q(\theta) d \theta+L \int_{s}^{\infty} v(\theta) d \theta\right] d s
\end{aligned}
$$




$$
\geq \quad-\frac{a_{2}-1}{a_{1}}-\frac{a_{2}-1}{3 a_{2}}=a
$$

implies that $\Psi x \in S$. Now for $x_{1}, x_{2} \in S$, we have

$$
\begin{aligned}
\left|\Psi x_{1}(t)-\Psi x_{2}(t)\right| & \leq \frac{1}{\left|p\left(\tau^{-1}(t)\right)\right|}\left|x_{1}\left(\tau^{-1}(t)\right)-x_{2}\left(\tau^{-1}(t)\right)\right| \\
& +\frac{K_{1}}{\left|p\left(\tau^{-1}(t)\right)\right|} \int_{T}^{\tau^{-1}(t)} \frac{1}{r(s)} \int_{s}^{\theta}\left|x_{1}(\sigma(\theta))-x_{2}(\sigma(\theta))\right| q(\theta) d \theta d s \\
& +\frac{K_{2}}{\left|p\left(\tau^{-1}(t)\right)\right|} \int_{T}^{\tau^{-1}(t)} \frac{1}{r(s)} \int_{s}^{\infty}\left|x_{1}(\eta(\theta))-x_{2}(\eta(\theta))\right| v(\theta) d \theta d s \\
& \leq \frac{1}{a_{2}}\left\|x_{1}-x_{2}\right\|+\frac{a_{2}-1}{3 a_{2}}\left\|x_{1}-x_{2}\right\| \\
& =\gamma\left\|x_{1}-x_{2}\right\|
\end{aligned}
$$

implies that

$$
\left\|\Psi x_{1}-\Psi x_{2}\right\| \leq \gamma\left\|x_{1}-x_{2}\right\|
$$

where $\gamma=\frac{1}{a_{2}}\left(1+\frac{a_{2}-1}{3}\right)<1$. Therefore, $\Psi$ is a contraction. Hence by the Banach's fixed point theorem $\Psi$ has a unique fixed point $x \in S$. It is easy to see that $\lim _{t \rightarrow \infty} x(t) \neq 0$. This completes the proof of the theorem.

\section{Discussion and Examples}

It is worth observation that we could succeed partially to establish the oscillation of all solutions of the nonlinear equation (1), when $|p(t)|<\infty$. We failed to obtain the necessary and sufficient conditions in the range $1 \leq p(t)<\infty$ and hence the undertaken problem is incomplete for all range of $p(t)$.

We conclude this section with the following examples to illustrate our main results:

\section{Example 3.1.}

$$
\left(x(t)-e^{-2 \pi} x(t-2 \pi)\right)^{\prime \prime}+e^{3 t} G(x(t-3 \pi))+e^{3 t+3 \pi} H(x(t-2 \pi))=0, t \geq 2 \pi,
$$

where $G(x)=H(x)=x^{3}$. All conditions of Theorem 2.6 are satisfied for (1). Hence, every solution of (1) either oscillates or converges to zero as $t \rightarrow \infty$. In particular, $x(t)=e^{-t}$ sint is one of such solution of (1). 


\section{Acknowledgments}

This work is supported by Rajiv Gandhi National Fellowship (UGC), New Delhi, India, through the letter No. F1-17.1/2013-14/RGNF-2013-14-SC-ORI42425, dated Feb. 6, 2014.

\section{References}

[1] R. P. Agarwal, M. Bohner, T. Li, C. Zhang, Oscillation of second order differential equations with a sublinear neutral term, Carpathian J. Math., 30(2014), 1-6.

[2] B. Baculikova, J. Dzurina, Oscillation theorems for second order neutral differential equations, Comput. Math. Appl., 61(2011), 94-99.

[3] B. Baculikova, J. Dzurina, Oscillation theorems for second order nonlinear neutral differential equations, Comput. Math. Appl., 62(2011), 4472-4478.

[4] B. Baculikova, T. Li, J. Dzurina, Oscillation theorems for second order neutral differential equations, Elect. J. Qual. Theo. Diff. Eqn., 74(2011), 1-13.

[5] J. K. Hale, Theory of functional differential equations, Springer-Verlag, New York, (1977).

[6] T. H. Hildebrandt, Introduction to the Theory of Integration, Academic press, New York, (1963).

[7] T. Li , Y. V. Rogovchenko, Oscillation theorems for second order nonlinear neutral delay differential eqquations, Abst. Appl. Anal., 2014(2014), ID 594190, 1-5.

[8] Y. Qian, R. Xu, Some new osciilation criteria for higher order quasi-linear neutral delay differential equations, Diff. Equ. Appl., 3(2011), 323-335.

[9] A. K. Tripathy, B. Panda, A. K. Sethi, On oscillatory nonlinear second order neutral delay differential equations, Diff. Equ. Appl., 8(2016), 247-258.

[10] Q. Yang, Z. Xu, Oscillation criteria for second order quasi-linear neutral delay differential equations on time scales, Comp. Math. Appl., 62(2011), 3682-3691.

[11] L. Ye, Z. Xu, Oscillation criteria for second order quasilinear neutral delay differential equations, Appl. Math. Comp., 207(2009), 388-396. 
